\title{
Investigation of stationary trajectories with associated milling by spur gears
}

\author{
Alexandra A. Gubanova* \\ Don State Technical University, Department of Automation of Production processes, 344000 \\ Rostov-on-Don Gagarin square 1, Russia
}

\begin{abstract}
When milling in a steady state, unlike, for example, turning, there are periodic elastic deformation displacements of the tool relative to the workpiece along the machining path. Instead of an equilibrium point, we consider a certain closed trajectory of elastic deformations. This is the trajectory to which all the trajectories approach asymptotically, while forces and deformations that mutually affect each other through the mechanism of changing the area of the cut-off layer are redistributed. The article proposes a mathematical apparatus and algorithms for calculating the trajectory of establishing a stationary state. The case of milling with spur mills is considered. A mathematical description of the "basic" dynamic model of the associated milling by the side teeth of the end mills for the complete non-stationary nonlinear connection formed by the milling process is presented. It differs from known models by considering spatial oscillations, taking into account the dependence of forces on the cutting speeds, taking into account the rates of elastic deformation displacements, taking into account the retardation of forces with respect to deformation displacements, and also by nonlinear damping caused by forces acting on the trailing edge of the tool. In addition, periodic changes in the parameters and the formation of the surface at the previous contact of the tooth are taken into account.
\end{abstract}

\section{Basic assumptions and generalized equation of dynamics for spur gears}

The complexity of studying the dynamics of milling lies in the fact that in the mathematical description of this process it is necessary to consider nonlinear equations with periodically changing coefficients, which are caused by the intermittence of the cutting process by each tooth of the milling cutter [1].

In this study, we make assumptions for the dynamic model of the milling process[2].

1. The angular velocity of the tool is unchanged and its torsional deformations are absent. This is true for most machines, since the power of the cutting process is an order of magnitude smaller than the power of the spindle rotation motor, and the mechanical part of the spindle together with the tool can be considered as an absolutely rigid body.

\footnotetext{
${ }^{*}$ Corresponding author: anatoliya81@mail.ru
} 
2. Tool deformations are considered in a plane normal to its axis, the workpiece is deformed in the same plane. The deformation displacement of the workpiece, considered at the point of contact with the tool, is indicated.

3. When studying dynamics, we confine ourselves to the basic forms of oscillations of the instrument. In this case, to describe the dynamics, the tool subsystem can be schematized as shown in Fig. 1.

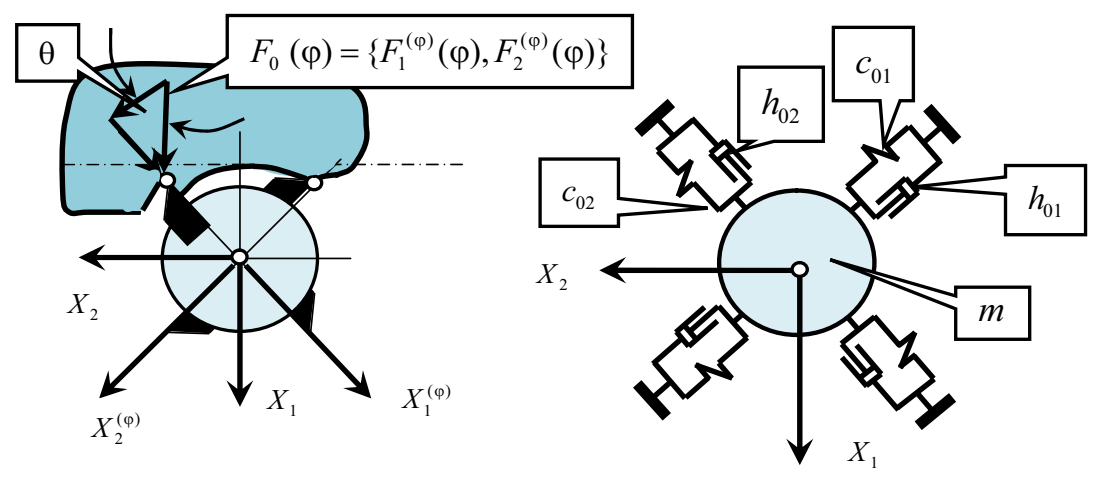

Fig. 1. Scheme of interaction between the tool subsystems and the workpiece being processed.

4. The forces depend on the area of the layer being cut, which varies depending on the angle of rotation of the tool and the elastic deformations. The cutting speed varies only due to changes in the speeds of the elastic deformation displacements of the tool in a plane normal to the axis of rotation of the tool. Since the milling of the end milling cutters with side cutting blades is considered, when milling with a straight milling cutter the width of the cut sheet remains unchanged. Therefore, the forces at a constant angular velocity depend only on the thickness of the cut-off layer.

5. The compliance of the tool in the plane is characterized by an ellipse, e.g. the elasticity property is linear and the elasticity matrix is symmetric[3], potential and positively definite. The tool itself will be considered as an absolutely solid body having a mass. Parameters of the tool subsystem while moving the table remain unchanged, the parameters of the subsystem of the workpiece can depend on them.

6. Processing with each blade can be considered as free turning with variable cutting depth. The orientation of forces with respect to the coordinates of the cutting blade is unchanged. Therefore, if the value of the strength modulus is given, then its projection onto the orthogonal coordinate system tied to the cutting blade is determined as

$$
F=\left[\begin{array}{l}
F_{1} \\
F_{2}
\end{array}\right]=\left[\begin{array}{cc}
\chi_{1} & 0 \\
0 & \chi_{2}
\end{array}\right]\left[\begin{array}{cc}
F_{0} & 0 \\
0 & F_{0}
\end{array}\right]
$$

where $F=\left\{F_{1}, F_{2}\right\}^{T}$ - vector-function of the cutting forces; $\chi_{1}, \chi_{2}$ are angular coefficients satisfying the normalization condition $\left(\chi_{1}\right)^{2}+\left(\chi_{2}\right)^{2}=1 ; F_{0}$ - a force module that depends on the modes $S_{P}^{(0)}, t_{P}^{(0)} V_{P}^{(0)}$ (longitudinal feed, depth and cutting speed) and elastic deformation displacement of the cutting blade along with the tool. The angular coefficients can be determined from the angle $\theta$ in Fig. 1. Thus, the forces depend on the coordinates of the system, and they represent the model of forces in these coordinates. Depending on the model of these forces, the properties of the system change. Therefore, an adequate description of the forces in the state coordinates is an important problem [4]. On the basis 
of the schematization (Figure 2), the equation of the dynamics of the system can be represented as

$$
\left\{\begin{array}{l}
m \frac{d^{2} X}{d t^{2}}+h \frac{d X}{d t}+c X=\sum_{i=1}^{i=k} F\left[S_{P}^{(0)}(t), t_{P}^{(0)}(t), V_{P}^{(0)}, X, \frac{d X}{d t}, Y, \frac{d Y}{d t}\right] \\
M(L) \frac{d^{2} Y}{d t^{2}}+H(L) \frac{d Y}{d t}+C(L) Y=\sum_{i=1}^{i=k} F\left[S_{P}^{(0)}(t), t_{P}^{(0)}(t), V_{P}^{(0)}, X, \frac{d X}{d t}, Y, \frac{d Y}{d t}\right]
\end{array}\right.
$$

where $m, M(L), h, H(L), c, C(L)$-positive definite symmetric matrices of inertial, dissipative and elastic coefficients of $2 \otimes 2$ size related to the subsystems of the tool and workpiece; $k$ - number of contact of the cutting blade with the workpiece.

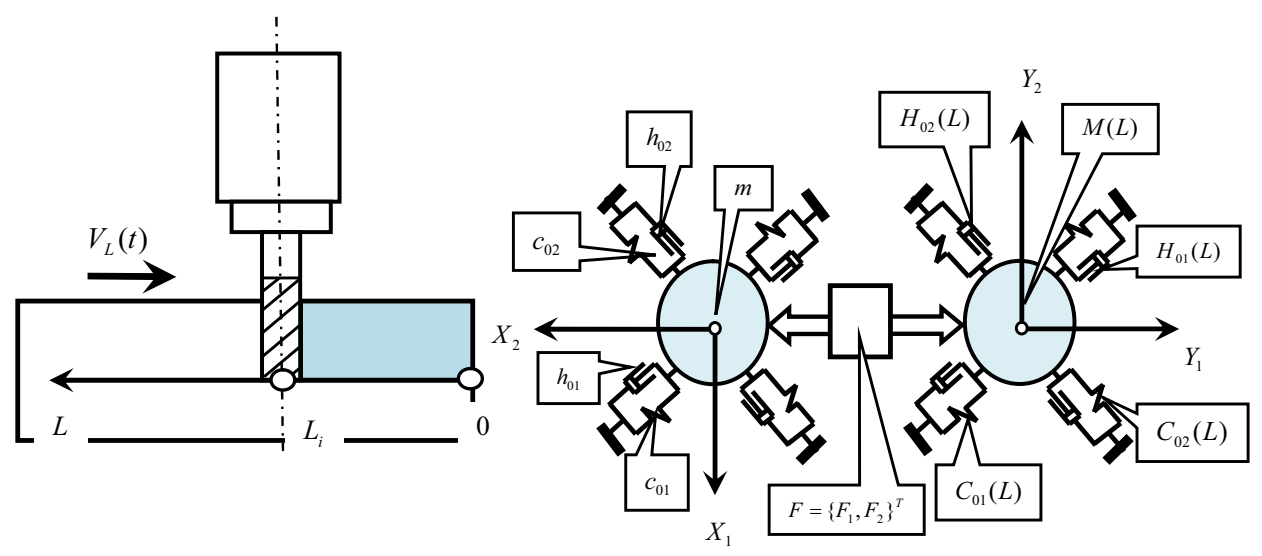

Fig. 2. Scheme of interaction between the tool subsystems and the workpiece being processed

On the right in (2) are the cutting forces represented in the functions of the state coordinates and technological regimes. It is believed that the cutter has $k$ cutting blades, and the forces acting on the workpiece and the tool are equal and opposite in sign. In the future, the functions defined in a general form $\sum_{i=1}^{i=k} F\left[S_{P}^{(0)}(t), t_{P}^{(0)}(t), V_{P}^{(0)}, X, \frac{d X}{d t}, Y, \frac{d Y}{d t}\right]$ will be revealed. When considering longitudinal milling, the feed to the cutter tooth is determined as

$$
S_{P}(t)=\int_{t-T}^{t}\left\{V_{L}(t)-v_{X_{2}}(t)-v_{Y_{2}}(t)\right\} d t
$$

where $V_{L}(t)$ - the speed of the table along the workpiece (Figure 2); $v_{X_{2}}(t), \quad v_{Y_{2}}(t)$ - the speed of elastic deformation displacements of the tool and workpiece in the direction of processing; $T$ - the time between the contacts of the teeth. Traditionally, the amount of feed per tooth corresponds to a steady value, when $V_{L}=$ const, and $v_{X_{2}}=0, v_{Y_{2}}=0$. Then it is clear from (3) that $S_{P}=V_{L} T$. If $\int_{t-T}^{t}\left\{v_{X_{2}}(t)+v_{Y_{2}}(t)\right\} d t \neq 0$ (this occurs when overlapping the contact of the teeth with the workpiece), then there is a section in which the deviation of the feed amount from its kinematic value $S_{P}=V_{L} T$ is observed. This is a part of the transition process in establishing a stationary state. Moreover, in the area of the transient process there is a redistribution of forces and elastic deformation displacements. In this 
case, the time $T$ between two successive tooth contacts changes; the current value of the technological allowance due to the permanent deformation of the tool and workpiece, determined by the fact that the forces averaged over the time are not equal to zero; amount of feed per tooth. These factors cause a self-regulation of the dynamic regime[5], which leads to the establishment of stationary trajectories of forces, deformations and variations in the allowance, attributable to each tooth of the mill.

Then

$$
\left\{\begin{array}{l}
c X^{*}=\sum_{i=1}^{i=k} F\left[S_{P}^{(0)}(t), t_{P}^{(0)}(t), V_{P}^{(0)}, X^{*}, 0, Y^{*}, 0\right] ; \\
C(L) Y^{*}=\sum_{i=1}^{i=k} F\left[S_{P}^{(0)}(t), t_{P}^{(0)}(t), V_{P}^{(0)}, X^{*}, 0, Y^{*}, 0\right] .
\end{array}\right.
$$

If we do not take into account the deformation of the workpiece, then (4) is transformed to the form of

$$
\left\{\begin{array}{l}
c_{1,1} X_{1}+c_{2,1} X_{2}=F_{1}\left[S_{P}^{(0)}(t), t_{P}^{(0)}(t), V_{P}^{(0)}, X_{1}, X_{2}\right] \\
c_{1,2} X_{1}+c_{2,2} X_{2}=F_{2}\left[S_{P}^{(0)}(t), t_{P}^{(0)}(t), V_{P}^{(0)}, X_{1}, X_{2}\right]
\end{array}\right.
$$

where $F_{1}, F_{2}$ - - projections of cutting forces on the axis $X_{1}, X_{2}$. To simplify the indexing here and in this chapter, the asterisks at coordinates are removed. The angle between the coordinate system $X_{1}, X_{2}$ and $\gamma_{1}, \gamma_{2}$ is denotedas $\alpha$. The transition from the system $X_{1}, X_{2}$ to $\gamma_{1}, \gamma_{2}$ is carried out with the help of the rotation operator:

$$
\begin{aligned}
& c=\left[\begin{array}{cc}
\cos \alpha & \sin \alpha \\
-\sin \alpha & \cos \alpha
\end{array}\right] \cdot\left[\begin{array}{cc}
c_{0,1} & 0 \\
0 & c_{0,2}
\end{array}\right] \cdot\left[\begin{array}{cc}
\cos \alpha & -\sin \alpha \\
\sin \alpha & \cos \alpha
\end{array}\right]= \\
& =\left[\begin{array}{cc}
\left(c_{0,1} \cos ^{2} \alpha+c_{0,2} \sin ^{2} \alpha\right) & {\left[\frac{1}{2}\left(c_{0,2}-c_{0,1}\right) \sin 2 \alpha\right]} \\
{\left[\frac{1}{2}\left(c_{0,2}-c_{0,1}\right) \sin 2 \alpha\right]} & \left(c_{0,1} \sin ^{2} \alpha+c_{0,2} \cos ^{2} \alpha\right)
\end{array}\right] .
\end{aligned}
$$

(6) shows that when $c_{0,1}=c_{0,2}$ the matrix $c$ is diagonal for any orthogonal system. The end mill has the property of symmetry, it can be regarded with sufficient approximation as a round rod. Therefore, the condition $c_{0,1}=c_{0,2}$ is satisfied. In this case, the matrix "c" is always diagonal. Moreover, bending vibrations of the end mill, as shown by experimental studies, have only frequencies that do not depend on the direction of excitation[6].

We emphasize that the end mill has the property of symmetry in the plane $X_{1}, X_{2}$. Therefore, its deformation displacements are always oriented along the direction of the external force. This is true, at least in the low-frequency range (up to $1.0-2.5 \mathrm{kHz}$ ). The symmetry of the elastic properties of the end mills allows us to make a conclusion: any orthogonal coordinate system whose origin coincides with the axis of rotation of the milling cutter in the plane normal to the axis of rotation of the milling cutter is the main one. Thus, you can always choose one of the axes, the direction of which corresponds to the direction of the cutting force. Then the deformation displacement of the tool will coincide with the direction of the force (Figure 3). Three coordinate systems can be distinguished (Figure 3): the first is oriented normally to the formed surface of the part $\left(X_{1}, X_{2}\right)$; the second - along the current angle of the cutting blade $\left(X_{1}^{(\varphi)}, X_{2}^{(\varphi)}\right)$; the third system, one of whose axes 
coincides with the orientation of the cutting force, reduced to the center of mass of the mill $\left(X_{1}^{(\varphi-\theta)}, X_{2}^{(\varphi-\theta)}\right)$. The first coordinate system is used to calculate the trace of the workpiece on the surface from the cutter and the trajectory of the cutting blade, taking into account the elastic deformation displacements; the second - to determine the forces, the value of which depends on the angle, technological modes and deformation of the cutter in the direction; the third - to calculate the deformation displacement in the direction of the application of force $[7,8]$. The relationship between deformation displacements in different systems is determined by expressions.

$$
\left\{\begin{array}{c}
X_{1}=X_{1}^{(\varphi-\theta)} \cos (\varphi-\theta) \\
X_{2}=-X_{1}^{(\varphi-\theta)} \sin (\varphi-\theta)
\end{array}\right.
$$

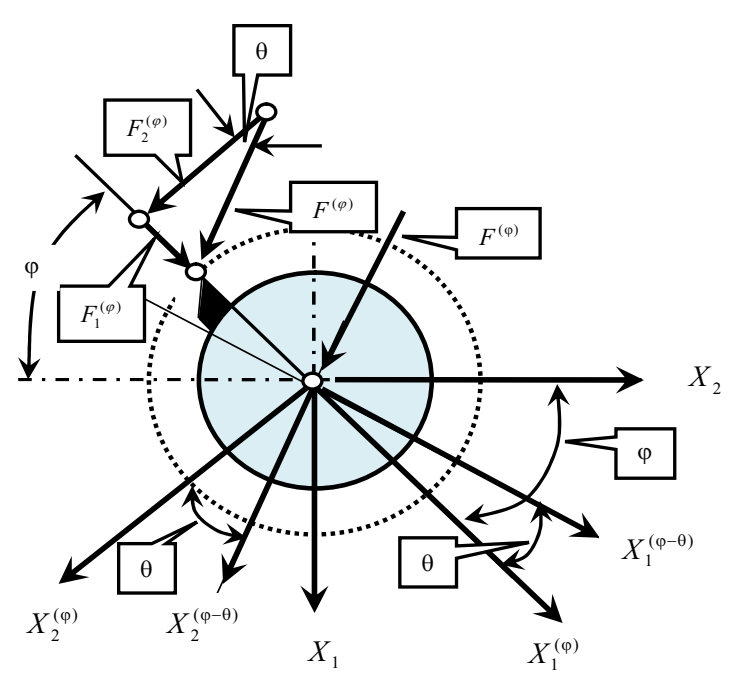

Fig. 3. Transformation of the orthogonal coordinate systems of deformation displacement of the tip of the cutting blade in a plane normal to the axis of rotation of the milling cutter.

$$
\left\{\begin{array}{l}
X_{1}^{(\varphi)}=X_{1}^{(\varphi-\theta)} \cos (\theta) \\
X_{2}^{(\varphi)}=-X_{1}^{(\varphi-\theta)} \sin (\theta)
\end{array}\right.
$$

Displacements $X_{1}^{(\varphi)}(t)$ do not affect the shear area, but their speed $d X_{1}^{(\varphi)}(t) / d t$ changes the cutting speed. Therefore, the equation of system dynamics considered with respect to the contact of one cutting blade with the workpiece in the coordinate system $\left(X_{1}^{(\varphi)}, X_{2}^{(\varphi)}\right)$ will be the following

$$
m \frac{d^{2} X^{(\varphi)}}{d t^{2}}+h \frac{d X^{(\varphi)}}{d t}+c X^{(\varphi)}=F\left\{S_{P}^{(0)}(t, \varphi),\left[t_{P}^{(0)}\left(X_{2}^{(\varphi)}(\varphi)-X_{1}^{(\varphi)}\right], V_{P}^{(0)}, \frac{d X_{1}^{(\varphi)}}{d t}, \frac{d X_{2}^{(\varphi)}}{d t}\right\},\right.
$$

where $X=\left\{X_{1}^{(\varphi)}, X_{2}^{(\varphi)}\right\}^{T} ; \quad m=\left[\begin{array}{cc}m & 0 \\ 0 & m\end{array}\right] ; \quad h=\left[\begin{array}{cc}h_{0} & 0 \\ 0 & h_{0}\end{array}\right] ; \quad c=\left[\begin{array}{cc}c_{0} & 0 \\ 0 & c_{0}\end{array}\right] . \quad$ In

$h_{0}=h_{1,0}=h_{2,0}$ and $c_{0}=c_{1,0}=c_{2,0}$. Since in this section we consider the equation of "slow" motions, it is necessary to analyze the change in forces and deformation displacements in the steady state for all angles, that is, consider the system 


$$
c X^{(\varphi)}(\varphi)=F\left\{S_{P}^{(0)}(\varphi),\left[t_{P}\left(\varphi, X_{2}^{(\varphi)}\right)-X_{1}^{(\varphi)}\right], V_{P}^{(0)}, 0,0\right\}
$$

Consequently, the trajectories change when passing from one tooth to another.

I express my gratitude to my scientific supervisor Zakovorotniy Vilor Lavrentievich for valuable advice in the planning of research and recommendations on the design of the article.

\section{Conclusion}

From the foregoing, in conclusion we can draw the following conclusions: due to the redistribution of forces and elastic deformation displacements, all the points of the beginning of the contact correspond to equal heights, i.e. there is no overlapping point of the contact. In the transitional regimes, the change in the current values of the allowance and the period of repetition of the elastic deformation displacements are noted. However, in the steady state (under the stationary steady state, we mean the state at which the functions of changing the deformation displacements repeat each other, that is, they are periodic functions), the trajectories of periodic elastic deformations characterize the same trajectory closed in space.

\section{References}

1. V. Zakovorotny, A. Gubanova, A. Lukyanov, Russian Engineering Research, 37 (2), 158 (2017)

2. S. Goldberg, American Journal of Physics, 35 (10), 934 (1967)

3. J. Hintikka, J. of Philosophical Logic, 41 (2), 505 (2012)

4. E. Budak, Y. Altintas, J. of Machine Tools and Manufacture, 35 (3), 459 (1995)

5. J. S. Tsai, C. L. Liao, J. of Materials Processing Technology, 94, 235 (1999)

6. H. Paris, G. Peigne, R. Mayer, J. of Machine Tools and Manufacture, 44 (15), 1567 (2004)

7. P. Kersting, D. Biermann, J. Machining Science and Technology, 13 (2), 196 (2009)

8. S. Voronov, I. Kiselev, J. of Multi-body Dynamics, 225 (3), 110 (2011) 